Luciana De Oliveira Marques ${ }^{1}$

Luiz Martins Collaço ${ }^{2}$

Larson Reichembach Pizzatto ${ }^{3}$

Beatriz Badinhani Mota Marcondes ${ }^{3}$

\title{
Efeitos da tibolona sobre o parênquima mamário: estudo experimental
}

\author{
Effects of tibolone on the breast parenchyma: experimental study
}

Artigo Original

Palavras-chave

Tibolona

Neoplasias da mama Terapia de reposição hormonal

Ratos

Estrogênios

Hiperplasia

Proliferação celular

Keywords

Tibolone

Breast neoplasms

Hormone replacement therapy

Rats

Estrogens/therapeutic use

Hyperplasia

Cell proliferation

\section{Resumo}

OBJETIVO: Avaliar o efeito da terapia hormonal com tibolona, em três períodos de tempo diferentes, sobre o tecido mamário de ratas castradas. MÉTODO: Foram utilizadas 60 ratas Wistar adultas e virgens, submetidas à ooforectomia. Após 21 dias de pós-operatório (PO), confirmado o hipoestrogenismo, os animais foram divididos aleatoriamente em 6 grupos: tibolona $1(n=10)$ recebeu tibolona $1 \mathrm{mg} /$ dia por 23 dias, tibolona $2(n=10)$, por 59 dias, tibolona $3(n=10)$, por 118 dias; os subgrupos controle $1(n=8)$, controle $2(n=7)$ e controle $3(n=10)$ receberam a água destilada por 23, 59 e 118 dias, respectivamente. Após o tratamento, foram ressecados seis pares de mamas, destinados à análise histológica pela coloração de hematoxilina e eosina (HE); o procedimento seguiu de eutanásia. Os parâmetros histológicos avaliados foram: hiperplasia epitelial e atividade secretora (AS). As variáveis foram submetidas à análise estatística, adotando-se como significante $\mathrm{p}<0,05$. RESULTADOS: Foram observadas alterações histológicas em 20/55 ratas, sendo: hiperplasia epitelial leve (HEB 1) em 7/55, hiperplasia epitelial moderada (HEB2) em 5/55, hiperplasia alvéolo-nodular (HAN) em 7/55, atipia sem proliferação epitelial em 1/55, não sendo encontrada hiperplasia severa (HEB3). Encontrou-se AS em 31 / 55 das ratas. A AS foi significativamente maior no grupo tibolona $(T)$, em todos os tempos avaliados $(p=0,001)$. As alterações histológicas analisadas não foram significantes comparando (p>0,05) os grupos controle (C) e T. A variável tempo de exposição à droga não apresentou significância, quando comparados os três períodos avaliados. CONCLUSÃO: Não foi verificada relação entre as alterações histológicas e a terapêutica com tibolona em curto, médio e longo prazo.

\section{Abstract}

OBJECTIVE: To assess the effect of tibolone on mammary tissue of castrated rats over 3 different periods of time. METHODS: Sixty virgin female Wistar rats were submitted to oophorectomy. Twenty-one days after surgery, with hypoestrogenism confirmed, the experimental rats were randomly assigned to six groups: Tibolone 1 ( $n=10$ ) received tibolone $1 \mathrm{mg} /$ day for 23 days, tibolone 2 ( $n=10$ ) for 59 days and tibolone $3(n=10)$ for 118 days. The groups control 1 ( $n=8)$, control 2 ( $n=7)$ and control 2 ( $n=10$ ) received distilled water for 23, 59 and 118 days, respectively. After treatment, all six pairs of mammary glands were removed and stained with hematoxylin and eosin (HE) for histological analysis after euthanasia. The histological parameters evaluated were: epithelial cell proliferation and secretory activity. The variables were analyzed statistically, with the level of significance set at 0.05. RESULTS: Histological changes were observed in 20/55 rats, mild epithelial hyperplasia in 7/55, moderate epithelial hyperplasia in 5/55, alveolar-nodular hyperplasia in 7/55, atypia without epithelial proliferation in 1/55, and no cases of severe epithelial hyperplasia were found. Secretory activity was observed in $31 / 55$ rats. The secretory activity was significantly higher in the tibolone groups compared to control at all the time points assessed $(p=0,001)$. The histological changes were did not show significance when the control and tibolone groups were compared. The time of exposure to tibolone did not show significance when the three different periods of evaluation were compared. CONCLUSION: No relation between histological modification and tibolone treatment was verified after short-, medium- and long-term treatment.
Correspondência

Luciana de Oliveira Marques Rua Padre Anchieta, 2.770 - Bigorrilho CEP: $80730-000$

Curitiba (PR), Brasil

Recebido

$16 / 03 / 2015$
Departamento de Experimentação Clínica e Cirúrgica, Curso de Medicina, Faculdade Evangélica do Paraná - FEPAR - Curitiba (PR), Brasil. 'Disciplina de Experimentação em Clínica e Cirúrgica, Curso de Medicina, Faculdade Evangélica do Paraná - FEPAR - Curitiba (PR), Brasil. 2Disciplinas de Histologia e Anatomia Patológica, Curso de Medicina, Faculdade Evangélica do Paraná - FEPAR; Disciplina de Anatomia Patológica, Curso de Medicina, Universidade Federal do Paraná - UFPR - Curitiba (PR), Brasil. ${ }^{3}$ Curso de Graduação em Medicina, Faculdade Evangélica do Paraná - FEPAR - Curitiba (PR), Brasil.

Conflito de interesses: não há 


\section{Introdução}

O climatério é o período da vida da mulher que marca o início do declínio da atividade ovariana e se estende até o encerramento completo de sua função hormonal. A experiência dessa fase é peculiar para cada mulher e difere de acordo com fatores hereditários, estilo de vida e cultura ${ }^{1}$.

Como a expectativa de vida na menopausa é de 32,7 anos, as mulheres passam mais de um terço de sua vida no estado de deficiência estrogênica pós-menopausal. Entre as mulheres que passam por sintomas climatéricos, $20 \%$ os descrevem como intoleráveis ${ }^{2}$.

Esse processo de adaptação fisiológica da vida da mulher vem sendo tratado desde a década de 1960 com formulações hormonais que visam suprir as deficiências da produção ovariana de estrógenos e controlar os sintomas climatéricos ${ }^{3}$.

A discussão envolvendo o papel da terapia hormonal da menopausa $(\mathrm{TH})$ na carcinogênese mamária é complexa $^{4}$. Entretanto, consideráveis evidências clínicas e epidemiológicas demonstram que a exposição contínua e cumulativa aos estrogênios elevam o risco de desenvolvimento de câncer mamário em 2 a 4 vezes s, $^{3,5,6}$.

Muitas pacientes que venceram o câncer de mama desenvolvem sintomas menopausais quando ingressam no período do climatério. Inibidores seletivos da recaptação de serotonina e outras intervenções não hormonais podem atenuar os sintomas, mas os resultados são pouco expressivos quando comparados ao tratamento com a TH convencional ${ }^{7}$. Nos casos de síndrome climatérica severa, a TH é a única que apresenta resultados satisfatórios no controle sintomatológico do climatério ${ }^{8}$.

A terapia estrogênica convencional é contraindicada em pacientes com risco de câncer de mama, dadas as chances de recidiva ${ }^{5,6}$. A tibolona é um esteroide sintético com características farmacológicas e clínicas diferentes dos hormônios sexuais convencionais. Hoje, muitas pacientes com um histórico de câncer de mama fazem uso de tibolona a fim de reduzir sintomas perimenopausais ${ }^{9-11}$. Entretanto, um histórico de câncer mamário continua sendo um alerta contra o uso da tibolona, apesar de não existirem evidências concretas confirmando o risco ${ }^{10}$.

A tibolona é rapidamente metabolizada pelo intestino e pelo fígado em seus três metabólitos ativos. Os isômeros alfa e beta apresentam ação estrogênica em vários tecidos. O isômero delta apresenta afinidade por receptores progestagênicos e androgênicos, mostrando, assim, efeito estrogênico restrito ${ }^{12}$. Os metabólitos estrogênicos agem em ossos e genitais, e, com os metabólitos androgênicos, aliviam os sintomas de "fogachos" e estimulam a energia e o bem-estar sexual. A estimulação do epitélio mamário é reduzida devido aos efeitos da tibolona sobre a ação das enzimas locais ${ }^{13}$.

Alguns autores e o grande estudo observacional Million Women Study (MWS) relatam que o uso de tibolona por até cinco anos esteve associado a um risco aumentado de câncer de mama ${ }^{14-16}$. Em contraste a isso, um grande estudo caso-controle não apresentou evidência de aumento do risco de câncer de mama, quando avaliado o tratamento exclusivo com tibolona por um período médio de 6,5 anos $^{17}$.

Além disso, dados recentes apontaram que a tibolona esteve associada a uma redução do risco de câncer de mama invasivo em um nível semelhante ao observado nos tratamentos com tamoxifeno e raloxifeno ${ }^{18}$, demonstrando inibição da aromatase em níveis superiores e efeitos agonistas sobre os receptores de estrogênios ${ }^{19}$.

A fim de esclarecer a real ação da tibolona sobre o parênquima mamário, o presente trabalho objetivou avaliar o efeito da TH com tibolona sobre a mama de ratas castradas em três períodos distintos de tratamento.

\section{Métodos}

O projeto foi aprovado pela Comissão de Ética em Pesquisa com Animais de Experimentação da Faculdade Evangélica do Paraná (FEPAR) sob o nº 004124/2013; foram seguidas todas as normas estabelecidas pelo Council for International Organization of Medical Sciences (CIOMS) e respeitados os preceitos da Sociedade Brasileira de Ciência em Animais de Laboratório.

O estudo experimental utilizou 60 ratos fêmeas virgens da linhagem Wistar (Rattus norvegicus albinus, Rodentia, Mammalia) com idade de 90 dias, oriundas do biotério central da FEPAR.

Os animais foram mantidos em gaiolas plásticas alojadas no biotério: grupos de cinco por gaiola. O local é equipado com ar-condicionado e sistema de exaustão para o controle da umidade, e a temperatura, mantida entre 22 e $24^{\circ} \mathrm{C}$. A iluminação artificial foi obtida por meio do uso de lâmpadas fluorescentes, sendo mantido um fotoperíodo de 12 horas claro (7-19h) e 12 horas escuro (19-7h). A alimentação foi oferecida ad libitum com água potável e ração específica para a espécie.

No primeiro momento do estudo, todas as ratas foram pesadas e, em seguida, ooforectomizadas bilateralmente, após anestesia geral, por intermédio da administração intraperitoneal de cetamina $10 \%$ na dose $10 \mathrm{mg} / 100 \mathrm{~g}$ de peso do animal e xilazina $2 \%$ na dose de $1 \mathrm{mg} / 100 \mathrm{~g}$. Após o procedimento, os animais receberam analgesia com cloridrato de tramadol de 12/12h via intramuscular (IM) no membro pélvico durante 3 dias. Posteriormente, os animais foram observados durante 21 dias, com a finalidade de esgotar os hormônios ovarianos endógenos circulantes 
e garantir o ingresso na fase de hipoestrogenismo, permitindo, assim, o repouso do tecido mamário ${ }^{20}$. Após esse período, foram realizadas coletas de esfregaços vaginais para análise citológica pela coloração de Papanicolau, como método indireto de confirmação do hipoestrogenismo, demonstrado pela atrofia ${ }^{21}$.

A análise das citologias vaginais foi realizada pela coloração de Papanicolau e sempre pelo mesmo patologista, sendo adotada a classificação na qual foram categorizadas as fases de Diestro, Pró-estro precoce, Pró-estro tardio e Estro. A fase de Diestro corresponde à predominância de leucócitos polimorfonucleares com pouco ou nenhum muco. A fase de Pró-estro precoce é caracterizada pela presença de leucócitos polimorfonucleares, células nucleadas e raras células epiteliais cornificadas presentes em um muco pegajoso. A fase de Pró-estro tardio é identificada pela presença exclusiva de células nucleadas. A fase de Estro é caracterizada pela presença única de células epiteliais cornificadas, exceto em estágios muito precoces, nos quais raras células nucleadas, formadas irregularmente, também podem ser visualizadas. $\mathrm{O}$ padrão normotrófico foi determinado pela presença de quantidades equivalentes de células superficiais e intermediárias. O padrão hipotrófico foi identificado por meio do predomínio das células intermediárias (70-100\%) em relação às células superficiais (0-30\%). O padrão hipertrófico foi caracterizado pelo predomínio de células superficiais (70-100\%) em relação às intermediárias (0-30\%).

A amostra foi dividida aleatoriamente em 2 grupos: um grupo tibolona $(\mathrm{T})$, com 30 ratas, para as quais foi administrada, via gavagem, tibolona $1 \mathrm{mg} / \mathrm{rata} / \mathrm{dia}$ diluída em $1 \mathrm{ml}$ de água destilada, e um grupo controle (C), também com 30 ratas, as quais receberam, por gavagem $^{22}, 1 \mathrm{ml}$ de água destilada. Cada grupo foi ainda dividido em 3 subgrupos de 10 ratas, conforme o tempo de tratamento, sendo: tibolona A (TA) durante 23 dias; tibolona B (TB) por 59 dias; e tibolona C (TC) durante 118 dias. O grupo C, por sua vez, foi subdividido da mesma forma em um subgrupo controle A (CA), um subgrupo controle $\mathrm{B}(\mathrm{CB})$ e um subgrupo controle $\mathrm{C}$ (CC), os quais receberam a água destilada por 23, 59 e 118 dias, respectivamente.

A medicação foi realizada em cada subgrupo durante três períodos diferentes equivalendo à $\mathrm{TH}$ da menopausa em curto, médio e longo prazo no humano. Como na fase adulta cada 1 ano humano equivale a 11,8 dias no rato, a TH em curto prazo foi realizada proporcionalmente a 2 anos para o humano, ou seja, 23 dias para o rato. A TH em médio prazo, realizada por 59 dias nas ratas, foi equivalente a 5 anos para o humano. A TH em longo prazo foi administrada às ratas por 118 dias, o equivalente a 10 anos no humano ${ }^{23}$.
Vinte e quatro horas após a última administração da tibolona em cada período de tratamento $(23,59$ e 118 dias), as ratas do subgrupo correspondente foram anestesiadas com a associação cetamina-xilazina da mesma forma e dose administradas anteriormente. Após a anestesia, foram removidos os seis pares de glândulas mamárias de cada animal. Em seguida, os materiais foram fixados em formalina tamponada e, após a fixação (24h), processados para a inclusão em parafina e destinados à confecção de lâminas para as análises histológicas do tecido mamário. Após o término do procedimento cirúrgico, foi realizada a eutanásia dos animais por meio da overdose anestésica, com os mesmos anestésicos e vias utilizados anteriormente.

A análise histológica do tecido mamário pela coloração de hematoxilina e eosina (HE) foi realizada em 4 campos por corte histológico, sob um aumento de 400 vezes, sempre pelo mesmo patologista. Foi adotado, nesta análise, um sistema de graduação histopatológico para anomalias epiteliais utilizado por Vicelli et al. ${ }^{20}$. Essa graduação se refere à hiperplasia epitelial e à atividade secretora (AS) nas células epiteliais, seguindo a seguinte classificação: normal, atipia sem proliferação epitelial; hiperplasia epitelial da unidade ductal terminal lobular leve (HEB1); hiperplasia epitelial da unidade ductal terminal lobular moderada (HEB2); hiperplasia epitelial da unidade ductal terminal lobular severa (HEB3); hiperplasia alvéolo-nodular (HAN), AS nas células epiteliais e análise do estroma mamário. Tal descrição tem sido adotada em diversos estudos que utilizam ratos como modelo experimental. A partir das alterações descritas na graduação adotada, e comparando-as com as observadas na mama humana, foi feita uma analogia, na qual: HEB1 corresponderia à hiperplasia ductal típica (HDT) leve; HEB2, à HDT moderada; HEB3, à hiperplasia ductal atípica (HDA). Considera-se a HDT leve sem risco para câncer invasor; a HDT moderada é considerada de baixo risco, duas vezes aumentado, para o câncer invasor; a HDA é considerada lesão associada a risco cinco vezes aumentado para o câncer invasor da mama ${ }^{24}$ (Quadro 1).

Para a comparação estatística dos grupos C e T em relação ao peso (inicial, final e diferença entre inicial e final), foi utilizado o teste $t$ de Student para amostras independentes. Para a comparação dos pesos inicial e final, dentro de cada grupo, foi usado o teste $t$ de Student

Quadro 1. Comparação das alterações mamárias humanas e murinas e grau de risco de doenca invasora

\begin{tabular}{|lcc|}
\hline Aliteração murina & Alteração humana & Risco de doença invasora \\
\hline HEB 1 & Hiperplasia ductal típica leve & Baixo risco \\
\hline HEB2 & Hiperplasia ductal típica moderada & Risco 2 vezes maior \\
\hline HEB3 & Hiperplasia ductal atíica & Risco 5 vezes maior \\
\hline
\end{tabular}

HEB: hiperplasia epitelial da unidade ducto-lobular terminal. 
para amostras pareadas. A fim de comparar os achados histológicos estatisticamente, os autores optaram por agrupar todas as alterações em um único grupo, sendo comparada a presença ou a ausência de tais alterações, sem distinção dos padrões histológicos supracitados. Para a comparação dos grupos e dos momentos de avaliação em relação à probabilidade de alteração histológica e à AS, foi considerado o teste exato de Fisher. Valores $p<0,05$ indicaram significância estatística. Os dados foram analisados com o programa computacional SPSS v. 20.0.

\section{Resultados}

$\mathrm{Na}$ análise final foi incluído neste estudo um total de 55 das 60 ratas que iniciaram o experimento. Tal fato ocorreu devido a 3 óbitos no pós-operatório (PO) imediato das ooforectomias, um óbito após 60 dias de experimento, não associado à administração da medicação, e a um caso de exclusão do experimento por trofismo vaginal incompatível com estado de hipoestrogenismo prévio ao tratamento.

A análise estatística comparativa do peso inicial dos animais entre todos os grupos apresentou um valor $\mathrm{p}>0,05$, o que demonstra a homogeneidade da amostra. Observou-se progressivo aumento de peso em todos os grupos durante os diferentes tempos do estudo ( $\mathrm{p}<0,001$ ). No entanto, apenas a diferença entre o peso inicial e o peso final dos animais submetidos ao tratamento durante 118 dias (C3 e T3) apresentou significância estatística $(\mathrm{p}<0,05)$. O grupo controle (C3) teve um ganho médio de peso de 99,5 g, valor significativamente maior em relação ao grupo $\mathrm{T}$ do mesmo período (T3), que apresentou um ganho médio de $51 \mathrm{~g}$.

$\mathrm{Na}$ análise de citologia vaginal observou-se que 59 ratas apresentavam padrão atrófico após 21 dias das ooforectomias, confirmando o hipoestrogenismo decorrente da castração. Apenas 1 das 60 ratas submetidas à castração cirúrgica permaneceu com trofismo vaginal ao exame citológico vaginal após 21 dias do procedimento. Tal rata foi submetida a uma segunda coleta que confirmou o achado de trofismo vaginal incompatível com o estado de hipoestrogenismo esperado, sendo, portanto, excluída do experimento.

Ao final dos 118 dias de experimento, 20 ratas apresentaram alterações epiteliais focais em suas glândulas mamárias, sendo: HEB1 em 7 casos (35\%), HEB2 em 5 casos (25\%), HAN em 7 casos (35\%), atipia sem proliferação epitelial em 1 caso (5\%). Não foi observado nenhum caso de HEB3 (Figura 1).

Nas 55 ratas incluídas no estudo, a AS foi observada em 31 animais $(56,6 \%)$, sendo que 2 pertenciam ao grupo C, e 29, ao grupo que recebeu tibolona durante os 3 períodos de experimento. Todos os animais submetidos à terapêutica com tibolona apresentaram AS confirmada histologicamente, com exceção de 1 caso no subgrupo T1 que obteve uma resposta negativa. Trinta e cinco ratas $(63,6 \%)$ apresentavam mamas normais ao final do estudo.

Ao se comparar as alterações histológicas verificadas nos grupos $\mathrm{C}$ e $\mathrm{T}$ em cada período do experimento, não foram encontrados dados significantes que possam atribuir as alterações presentes no grupo $\mathrm{T}$ à terapêutica com a droga em nenhum dos períodos avaliados no estudo (Tabela 1).

Para os animais submetidos ao experimento durante 23 dias (C1 e T1), foram observadas alterações histológicas em $4(22,2 \%)$ das 18 ratas analisadas no período, sendo todas as alterações histológicas verificadas no grupo que recebeu tibolona. Foram encontrados 1 caso de HEB 1 (25\%), 2 casos de HAN (50\%) e 1 caso enquadrado no padrão de atipia plana (25\%).

Nos animais analisados durante o período de 59 dias (C2 e T2) foram encontradas alterações em 4 (23,5\%) das 17 ratas. Das alterações encontradas, duas foram observadas no subgrupo controle (C2) e duas no subgrupo que recebeu tibolona (TB), sendo todas elas classificadas como HEB1.

Para o grupo de animais acompanhado durante o período de 118 dias (C3 e T3), foram observadas alterações histológicas em 12 (60\%) das 20 ratas. Entre todas as alterações encontradas, seis foram verificadas no subgrupo controle (C3) e seis observadas no subgrupo que recebeu tibolona pelo mesmo período (T3). No subgrupo C estiveram presentes 1 caso HEB1 (8,33\%) e 5 alterações HEB2 (41,6\%). Já no subgrupo tibolona foi encontrada uma alteração $(8,33 \%)$, classificada como HEB 1, e 5 casos $(41,6 \%)$ do padrão HAN.

Ao se comparar os três períodos de estudo no grupo $C$, não foram encontradas relações significantes entre o intervalo de tempo e a presença de alterações histológicas quando os animais foram avaliados sob um intervalo de até 60 dias. Entretanto, ao se comparar os períodos 1 (23 dias) e 3 (118 dias), foi encontrada significância $(p=0,013)$ que sugere que o avanço na idade esteve associado ao aumento de alterações histológicas, quando considerado um intervalo de tempo de 90 dias (Tabela 2).

Ao analisarmos os animais submetidos à terapêutica com tibolona, o maior tempo de exposição à droga não esteve associado ao aumento da incidência de alterações mamárias murinas, quando considerados intervalos de tempo de até 90 dias de TH com tibolona (Tabela 2).

A tibolona esteve associada ao aumento da AS em todos os períodos de tratamento avaliados neste estudo $(\mathrm{p}<0,001)$; tal fato já era esperado, uma vez que a ação hormonal progestagênica da tibolona sobre a mama apresenta relação direta com o aumento da AS mamária. Além disso, tais dados confirmam a absorção e a circulação sistêmica da droga durante todos os momentos avaliados no experimento. 

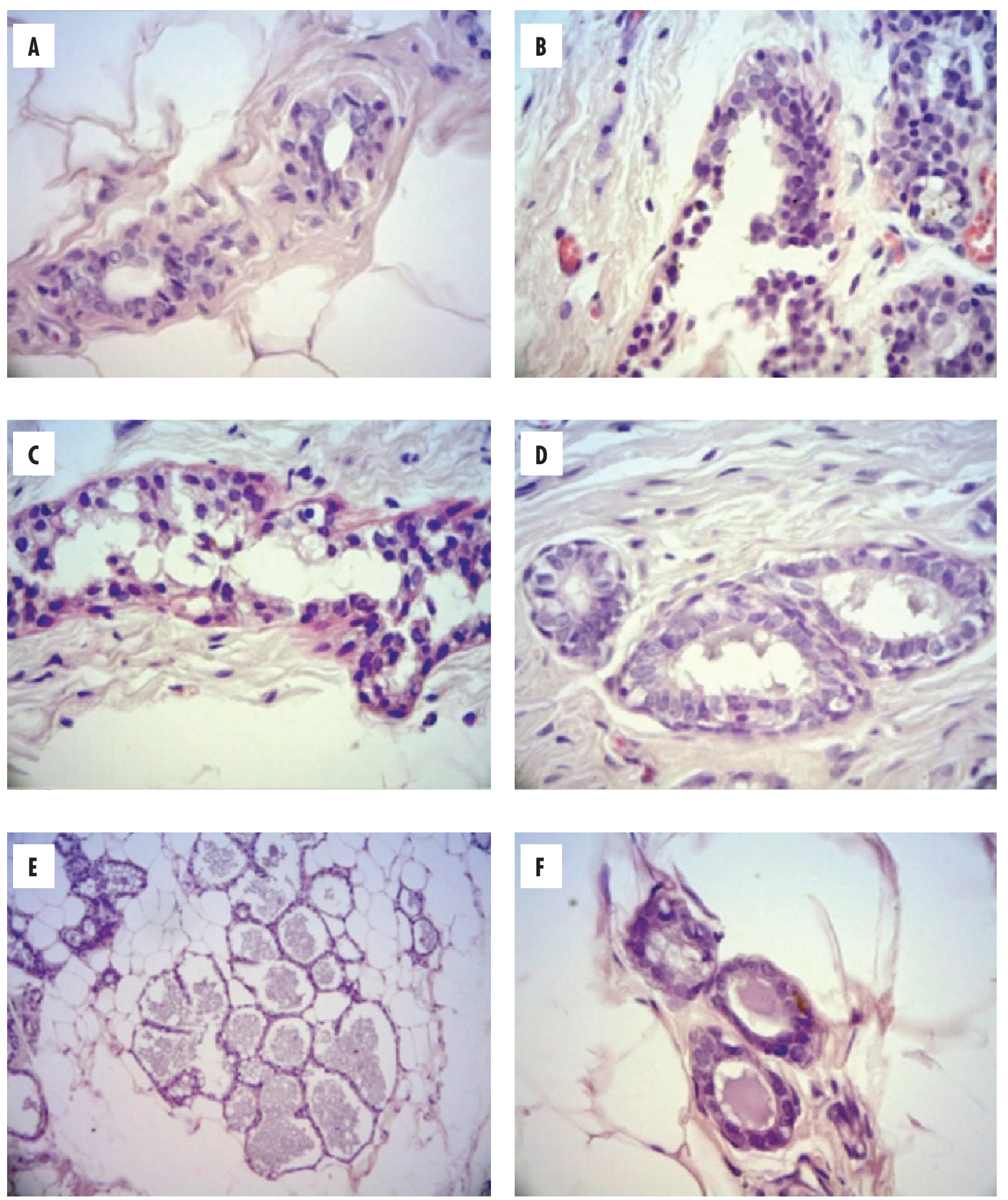

Figura 1. Fotomicrografia de tecido mamário nos grupos de estudo. (A) Ductos mamários com aspecto normal (hematoxilina e eosina 400x). (B) Hiperplasia epitelial da unidade ducto-lobular terminal leve em rata do grupo controle (hematoxilina e eosina 400x). (C) Hiperplasia epitelial da unidade ducto-lobular terminal moderada em rata do grupo tibolona (hematoxilina e eosina 400x). (D) Atipia plana em rata do grupo tibolona (hematoxilina e eosina 400x). (E) Hiperplasia alvéolo-nodular em rata do grupo tibolona (hematoxilina e eosina 100x). (F) Atividade secretora em rata do grupo tibolona (hematoxilina e eosina 400x) 
Tabela 1. Comparação das alterações histológicas entre os grupos controle e tibolona

\begin{tabular}{|c|c|c|c|c|c|c|c|c|c|c|c|c|}
\hline \multirow{3}{*}{ Avaliação histológica } & \multicolumn{4}{|c|}{ Período 1} & \multicolumn{4}{|c|}{ Período 2} & \multicolumn{4}{|c|}{ Período 3} \\
\hline & \multicolumn{2}{|c|}{ Controle 1} & \multicolumn{2}{|c|}{ Tibolona 1} & \multicolumn{2}{|c|}{ Controle 2} & \multicolumn{2}{|c|}{ Tibolona 2} & \multicolumn{2}{|c|}{ Controle 3} & \multicolumn{2}{|c|}{ Tibolona 3} \\
\hline & n & $\%$ & n & $\%$ & n & $\%$ & n & $\%$ & n & $\%$ & n & $\%$ \\
\hline Alteração & 0 & 0 & 4 & 40 & 2 & 29 & 2 & 20 & 6 & 60 & 6 & 60 \\
\hline Total & 8 & 100 & 10 & 100 & 7 & 100 & 10 & 100 & 10 & 100 & 10 & 100 \\
\hline Valor p: 0,091 & & \multicolumn{3}{|c|}{ Valor p: 1} & \multicolumn{4}{|c|}{ Valor p: 1} & & & \multicolumn{2}{|c|}{ Valor p: 1} \\
\hline
\end{tabular}

Tabela 2. Comparação entre os três períodos de estudo nos grupos controle e tibolona

\begin{tabular}{lcc}
\hline \multirow{2}{*}{ Períodos } & Controle & Tibolona \\
\cline { 2 - 3 } & Valor $p$ & Valor $p$ \\
\hline 23 dias versus 59 dias & 0,2 & 0,6 \\
23 dias versus 118 dias & 0,01 & 0,6 \\
59 dias versus 118 dias & 0,3 & 0,1 \\
\hline
\end{tabular}

\section{Discussão}

Apesar das claras diferenças em relação à espécie humana, os modelos experimentais induzidos em ratos constituem os mecanismos mais usados atualmente para o estudo do câncer de mama humano. Isso se deve à alta susceptibilidade por parte das glândulas mamárias de ratas em desenvolver lesões mimicamente similares às neoplasias mamárias no humano. Além disso, apresentam um mecanismo carcinogênico similar, no qual a origem da lesão se apresenta na exata localização da gênese na mama humana ${ }^{24,25}$.

Por meio de uma análise comparativa é possível avaliar os efeitos da terapêutica com tibolona sobre a atividade proliferativa das células ductais, bem como quantificar o risco de doença invasora associado à droga ${ }^{20}$.

O papel da tibolona sobre o tecido mamário tem sido considerado controverso nas últimas décadas. O fármaco é um esteroide sintético com um perfil diferente dos hormônios convencionais ${ }^{9,26}$. Acredita-se que a tibolona possa agir de maneira a contrariar efeitos estrogênicos no tecido mamário, exercendo uma ação de inibição tumoral ${ }^{12}$.

Existem vários mecanismos que possivelmente justifiquem a ação de inibição tumoral da tibolona. Um efeito antagonista dos hidroximetabólitos sobre os receptores de estrogênio associado à inibição da aromatase local no parênquima mamário já foi relatado ${ }^{27}$. Pesquisas mais recentes indicaram que a tibolona possui um papel inibitório na conversão do sulfato de estrogênio em estrogênio, por intermédio da inibição da enzima sulfatase ${ }^{28}$. A droga vem sendo associada a uma redução na taxa de proliferação das células epiteliais mamárias e ao favorecimento dos mecanismos de apoptose das células tumorais ${ }^{19}$. Um estudo recente que avaliou perfis de expressão genética de proliferação epitelial em macacos tratados com altas doses de tibolona encontrou um perfil transcripcional de proliferação no grupo T muito semelhante ao grupo C. Em tal estudo também não foi observada elevação na expressão de marcadores específicos de proliferação dos genes Ki-67 e KRT19 nos animais tratados com a droga. O subgrupo tibolona também não apresentou marcadores associados à sinalização de proliferação epitelial por fatores de crescimento ${ }^{29}$. Tais resultados são consistentes com outros trabalhos que não observaram elevações nos marcadores de expressão do gene $\mathrm{Ki}-67^{30} \mathrm{e}$ no risco de câncer mamário associadas ao uso de tibolona ${ }^{19}$.

O estudo LIBERATE propõe ao fármaco uma ação agonista ao estrogênio, contradizendo a hipótese da ação antagonista que os hidróximetabólitos teriam sobre os receptores estrogênicos $(\mathrm{RE})^{10}$. Entretanto, ao se avaliar os marcadores genéticos de atividade dos RE, foi observado um modesto efeito estrogênico por parte da tibolona, apesar de não terem sido encontrados marcadores de atividade progestagênica em nível nuclear ${ }^{29}$.

O uso de tibolona em pacientes com um histórico de câncer de mama esteve, porém, associado a uma elevação nas taxas de recorrência de câncer mamário em alguns outros estudos $^{16,31}$. O MWS também encontrou uma correlação importante entre o risco de câncer de mama e o uso de tibolona. Para mulheres que utilizaram tal terapêutica, o risco relativo (RR) de câncer foi de $1,45^{14}$. Contudo, existem algumas limitações importantes no que diz respeito à avaliação da tibolona neste estudo. Primeiramente, o regime anterior de TH foi alterado para tibolona em um terço das mulheres analisadas, sem qualquer justificativa. Sendo assim, a terapêutica prévia e os fatores de risco preexistentes, como maior densidade do parênquima mamário, não eram conhecidos nas mulheres que passaram a ser tratadas com tibolona. Outro ponto é que pode ter ocorrido viés de seleção, uma vez que o tempo médio decorrido entre a inclusão no estudo e o diagnóstico de câncer de mama foi de 1,2 anos, e de 1,7 anos entre a inclusão no estudo e o falecimento por câncer de mama. Tais resultados são pouco plausíveis do ponto de vista biológico ${ }^{32}$. Segundo a curva de Gompertz, uma célula tumoral leva 180 dias para se dividir, de forma que para se dividir 30 vezes levaria 10 anos, atingindo então $1 \mathrm{~cm}^{33}$. Dessa forma, seria improvável afirmar uma correlação direta entre a terapia com tibolona e o risco aumentado de câncer de mama em um período de tempo tão curto como o observado no MWS ${ }^{32,33}$. 
Em contraponto a grandes estudos contrários à reposição hormonal com tibolona, outras análises encontraram resultados que apoiam o seu emprego na terapêutica da menopausa. Vicelli et al. ${ }^{20}$, em um estudo experimental, não encontraram aumento na incidência de alterações histológicas nas mamas do subgrupo tibolona em relação ao controle. De forma semelhante, os dados encontrados neste estudo não revelaram alterações proliferativas significantes em nenhum dos intervalos de tratamento avaliados. Kubista et al. ${ }^{34}$ também demostraram que a tibolona não apresentou efeito sobre a proliferação celular em pacientes com câncer de mama primário ER positivo. Já diretamente sobre as células tumorais a droga esteve associada à elevação nos índices de marcadores de apoptose e à redução da atividade proliferativa dessas células ${ }^{35}$.

Um trabalho recente encontrou uma relação direta entre a duração da TH e o aumento no risco de câncer mamário ${ }^{16}$. Entretanto, neste estudo, não foram encontrados dados que confirmem que o maior tempo de exposição à droga seria responsável por um aumento das alterações histológicas no grupo T. Contudo, seria interessante ressaltar que, ao avaliarmos o período de 118 dias, as alterações encontradas no grupo $C$ pertenciam predominantemente ao padrão histológico HEB2. Já as alterações encontradas no grupo $\mathrm{T}$ eram representadas, em sua maioria, pelo padrão HAN, o qual tem sido associado a um pior prognóstico em relação ao risco de doença invasora por alguns autores ${ }^{20,36}$.

Apesar de alguns estudos negarem uma possível associação progressiva entre as lesões neoplásicas e o padrão $\mathrm{HAN}^{37}$, estudos mais recentes passaram a interpretar essas alterações como potenciais lesões precursoras de carcinoma mamário, uma vez que encontraram uma associação significante entre hiperplasia nodular e doença invasora em ratos tratados com $\mathrm{DMBA}^{36}$.

A metanálise de Formoso et al.$^{38}$ confirmou a eficácia e a segurança da droga em curto prazo. Em conformidade, neste trabalho experimental, não foram encontrados dados que vinculem as alterações histológicas identificadas no grupo avaliado em curto prazo à terapêutica com tibolona. No entanto, ao se avaliar o tempo de exposição à droga, os principais resultados disponíveis na literatura ainda não alcançaram um consenso em relação ao período seguro de contato com tal medicamento ${ }^{10,11,13,38}$.

Sendo assim, ao analisarmos todos os pontos levantados neste estudo, concluímos que a tibolona não apresentou relação com alterações proliferativas mamárias tanto em curto quanto em médio e longo prazo. Além disso, houve estímulo da AS mamária após o tratamento com tibolona em todos os períodos avaliados no estudo. Os dados disponíveis na literatura atualmente ainda apresentam informações que falham em confirmar a segurança da TH com tibolona. Dessa forma, ainda são necessários estudos de análise imuno-histoquímica e molecular que avaliem uma amostragem maior, a fim de esclarecer de forma definitiva a real ação de tal medicamento sobre o parênquima mamário.

\section{Agradecimentos}

Ao Conselho Nacional de Desenvolvimento Científico e Tecnológico (CNPq), pelo apoio financeiro por meio do Programa Institucional de Bolsas de Iniciação Científica (PIBIC).

\section{Referências}

1. Capote Bueno MI, Segredo Peréz AM, Gómez Zayas O. Climaterio y menopausia. Rev Cubana Med Gen Integr. 201 1;27(4):543-57.

2. El-Haij Fuleihan $G$. Tibolone and the promise of ideal hormonereplacement therapy. N Engl J Med. 2008;359(7):753-5.

3. Saether S, Bakken K, Lund E. The risk of breast cancer linked to menopausal hormone therapy. Tidsskr Nor Logeforen. 2012;132(1 1):1330-4.

4. Dinger JC, Heinemann LA, Möhner S, Thai DM, Assmann A. Breast cancer risk associated with different HRT formulations: a registerbased case-control study. BMC Womens Health. 2006;6:13.

5. Russo J, Russo $\mathrm{IH}$. The role of estrogen in the initiation of breast cancer. J Steroid Biochem Mol Biol. 2006; 102(1-5):89-96.

6. The North American Menopause Society. The 2012 hormone therapy position statement of the North American Menopause Society. Menopause. 2012;19(3):257-71.

7. Chen WY. Postmenopausal hormone therapy and breast cancer risk: current status and unanswered questions. Endocrinol Metab Clin North Am. 2011 ;40(3):509-18.
8. Pardini D. Terapia hormonal da menopausa. Arq Bras Endocrinol Metab. 2007;51(6):938-42.

9. Santen R, Stuenkel CA, Burger HG, Manson JE. Competency in menopause management: whither goest the internist? J Womens Health (Larchmt). 2014;23(4):281-5.

10. Kenemans P, Bundred NJ, Foidart JM, Kubista E, von Schoultz $B$, Sismondi $P$, et al. Safety and efficacy of tibolone in breast-cancer patients with vasomotor symptoms: a double-blind, randomised, non-inferiority trial. Lancet Oncol. 2009; 10(2): 135-46.

11. Morais-Socorro M, Cavalcanti MA, Martins R, Freire-Neto FP, Rezende AA, Azevedo GD, et al. Safety and efficacy of tibolone and menopausal transition: a randomized, double-blind placebocontrolled trial. Gynecol Endocrinol. 2012;28(6):483-7.

12. Guzmán-Silva MA, Pollastri CE, Andrade GM, Pantaleão JAS, Silva $\Perp$. Ação da tibolona no parênquima mamário. Femina. 2010;38(1):47-52.

13. Huang KE, Baber R; Asia Pacific Tibolone Consensus Group. Updated clinical recommendations for the use of tibolone in Asian women. Climacteric. 2010;13(4):317-27. 
14. Million Women Study Collaborators. Patterns of use of hormone replacement therapy in one million women in Britain, 1996-2000. BJOG. 2002;109(12):1319-30.

15. Stute P. Is breast cancer risk the same for all progestogens? Arch Gynecol Obstet. 2014;290(2):207-9.

16. Cordina-Duverger E, Truong T, Anger A, Sanchez M, Arveux $P$, Kerbrat $P$, et al. Risk of breast cancer by type of menopausal hormone therapy: a case-control study among post-menopausal women in France. PLoS One. 2013;8(1 1):e78016.

17. Beral V; Million Women Study Collaborators. Breast cancer and hormone-replacement therapy in the Million Women Study. Lancet. 2003;362(9382):419-27.

18. Callejo J, Cano A, Medina M, Villaronga M, Gonzalez-Bosquet $\mathrm{E}$, Sabria J, et al. Hormonal environment in the induction of breast cancer in castrated rats using dimethylbenzanthracene: influence of the presence or absence of ovarian activity and of treatment with estradiol, tibolone, and raloxifene. Menopause. 2005; 12(5):601-8.

19. Cummings SR, Ettinger B, Delmas PD, Kenemans P, Stathopoulos $V$, Verweii $P$, et al. The effects of tibolone in older postmenopausal women. N Engl J Med. 2008;359(7):697-708.

20. Vicelli JT, Gurgel MSC, Alvarenga M. Histologia mamária após uso de esteróides sexuais-estudo em ratas. Rev Assoc Med Bras. 2006;52(5):369-74.

21. Torres SMPS, Simões RS, Baracat MCP, Gomes RCT, Soares Júnior $J M$, Carbonel AAF, et al. Histomorfometria da mama de ratas tratadas com estrogênio e/ou progestagênio. Rev Assoc Med Bras. $2011 ; 57(2): 177-81$.

22. Henriques HN, de Carvalho AC, Soares Filho PJ, Pantaleão JA, GuzmánSilva MA. Effect of prolonged use of high dose of tibolone on the vagina of ovariectomized rats. Int J Exp Pathol. 2011 ; 92(4):266-71

23. Andreollo NA, Santos EF, Ar aújo MR, Lopes LR. Idade dos ratos versus idade humana: qual é a relação? $A B C D$ Arq Bras Cir Dig. $2012 ; 25(1): 49-51$.

24. Costa I, Solanas M, Escrich E. Histopathological characterization of mammary neoplastic lesions induced with 7,12 dimethylbenz $(\alpha)$ anthracene in the rat: a comparative analysis with human breast tumors. Arch Pathol Lab Med. 2002;126(8):915-27.

25. Haslam SZ. Experimental mouse model of hormonal therapy effects on the postmenopausal mammary gland. Breast Dis. 2005-2006;24(1):71-8.
26. Lambrinoudaki I. Progestogens in postmenopausal hormone therapy and the risk of breast cancer. Maturitas. 2014;77(4):311-7.

27. Ederveen AG, Kloosterboer HJ. Tibolone exerts its protective effect on trabecular bone loss through the estrogen receptor. J Bone Miner Res. 2001;16(9):1651-7.

28. de Gooyer ME, Deckers GH, Schoonen WG, Verheul HA, Kloosterboer $\mathrm{HJ}$. Receptor profiling and endocrine interactions of tibolone. Steroids. 2003;68(1):21-30.

29. Wood CE, Branstetter D, Jacob AP, Cline JM, Register TC, Rohrbach $\mathrm{K}$, et al. Progestin effects on cell proliferation pathways in the postmenopausal mammary gland. Breast Cancer Res. $2013 ; 15(4):$ :R62.

30. Cline JM, Register TC, Clarkson TB. Effects of tibolone and hormone replacement therapy on the breast of cynomolgus monkeys. Menopause. 2002;9(6):422-9.

31. Bundred NJ, Kenemans P, Yip CH, Beckmann MW, Foidart $J M$, Sismondi $P$, et al. Tibolone increases bone mineral density but also relapse in breast cancer survivors: LIBERATE trial bone substudy. Breast Cancer Res. 2012;14(1):R13.

32. Erel CT, Senturk LM, Kaleli S. Tibolone and breast cancer. Postgrad Med J. 2006;82(972):658-62

33. Domingues JS. Análise do modelo de Gompertz no crescimento de tumores sólidos e inserção de um fator de tratamento. Biomatemática. 2011;21:103-12.

34. Kubista E, Planellas Gomez JV, Dowsett M, Foidart JM, Pohlodek $K$, Serreyn R, et al. Effect of tibolone on breast cancer cell proliferation in postmenopausal ER+ patients: results from STEM trial. Clin Cancer Res. 2007;13(14):4185-90.

35. Lambrinoudaki I, Karaflou M, Kaparos G, Alexandrou A, Creatsa $M$, Aravantinos $L$, et al. Effect of tibolone and raloxifene on serum markers of apoptosis in postmenopausal women. Climateric. 2013;16(2):258-64

36. Thompson JH, Singh M. Rat models of premalignant breast disease. J Mammary Gland Biol Neoplasia. 2000;5(4):409-20.

37. Purnell DM. The relationship of terminal duct hyperplasia to mammary carcinoma in 7,12-dimethylbenz(a)anthracene-treated LEW/Mai rats. Am J Pathol. 1980;98(2):31 1-24.

38. Formoso G, Perrone E, Maltoni S, Balduzzi S, D'Amico R, Bassi C, et al. Short and long term effects of tibolone in postmenopausal women. Cochrane Database Syst Rev. 2012;2:CD:008536. 